\title{
STRESS FUNCTIONS FOR COUPLE AND DIPOLAR STRESSES*
}

\author{
By D. E. CARLSON, (University of Illinois, Urbana, Illinois)
}

1. Introduction. In a recent paper Gurtin [5] established a complete representation for symmetric second-order tensor fields in terms of another symmetric second-order tensor field and a vector field. His representation is analogous to the Stokes-Helmholtz resolution of a vector field into the curl of another vector field and the gradient of a scalar field. Gurtin was thus led to a complete representation for equilibrated stress fields in continuum mechanics-the generalized Beltrami solution. It is the purpose of the present paper to extend these results to more general theories of continuum mechanics, namely the Cosserat [1] continuum and the dipolar continuum of Green and Rivlin [6].

Günther [3] has obtained a stress function solution of the Cosserat equations of equilibrium. However, his solution cannot be complete for regions with holes because in the absence of couple stresses, it reduces to the Beltrami solution (cf. Gurtin [5]).

Sections 2 and 3 contain the equilibrium equations of the above theories and several solutions in terms of stress functions. In Section 4, following Gurtin [5], we establish some general representation theorems for second- and third-order tensor fields and utilize them to demonstrate the completeness of the solutions presented in Sections 2 and 3.

2. Couple stresses. The stress equations of equilibrium for a Cosserat [1] continuum may be written as

$$
\begin{aligned}
t_{i i, i}+f_{j} & =0 \\
m_{i j, i}+e_{j k l} t_{k l}+c_{i} & =0
\end{aligned}
$$

when referred to rectangular Cartesian coordinates.** In Eqs. (2.1) $t_{i j}$ is the stress tensor, $m_{i j}$ the couple stress tensor, $f_{i}$ the body force per unit volume, and $c_{i}$ the body couple per unit volume.

Introducing the quantities

$$
\begin{aligned}
m_{i j k} & =\frac{1}{2} e_{l i k} m_{i l} \quad\left(m_{i j}=e_{i m n} m_{i m n}\right), \\
c_{i j} & =\frac{1}{2} e_{l i j} c_{l} \quad\left(c_{i}=e_{i m n} c_{m n}\right),
\end{aligned}
$$

we can write Eqs. (2.1) in the equivalent form

$$
\begin{aligned}
t_{i j, i}+f_{i} & =0, \\
m_{i j k, i}+t_{[j k 1}+c_{i k} & =0 .
\end{aligned}
$$

Note from (2.2) that

$$
m_{i(j k)}=0, \quad c_{(i j)}=0 .
$$

*Received June 18, 1965.

${ }^{* *}$ We use the usual indicial notation of Cartesian tensor analysis. Latin subscripts have the range $(1,2,3)$, and summation over repeated subscripts is implied. Subscripts preceded by a comma indicate differentiation with respect to the corresponding Cartesian coordinate. Kronecker's delta and the alternating symbol are denoted by $\delta_{i j}$ and $e_{i j k}$, respectively. A pair of subscripts is enclosed by parentheses ( ) to indicate the symmetric part and by brackets [ ] to indicate the skewsymmetric part. 
We will work with $m_{i j}$ and Eqs. (2.1). However, we can immediately write our results in terms of $m_{i j k}$ by use of Eqs. (2.2).

The following theorems, which provide solutions to Eqs. (2.1), may be confirmed by direct substitution. We choose not to include the obvious smoothness requirements in the statements of these theorems.

Theorem 2.1. Let $\vartheta_{i}, \varphi_{i}, \theta_{i}$, and $\phi_{i}$ satisfy

$$
\begin{aligned}
\nabla^{2} \vartheta_{i}+e_{i j k} \varphi_{i, k} & =-f_{i}, \\
\nabla^{2} \theta_{i}+e_{i j k} \phi_{i, k}+2 \varphi_{i} & =-c_{i} .
\end{aligned}
$$

Define $t_{i j}$ and $m_{i j}$ by

$$
\begin{aligned}
t_{i j} & =e_{i m p} e_{i n q} \omega_{m n, p q}-\delta_{i j} \vartheta_{k, k}+\vartheta_{i, j}+\vartheta_{i, i}+e_{i j k} \varphi_{k}, \\
m_{i j} & =e_{i m p} e_{i n q} \Omega_{m n, p q}-\delta_{i j} \theta_{k, k}+\theta_{i, i}+\theta_{i, i}+e_{i j k} \phi_{k},
\end{aligned}
$$

where $\omega_{i i}=\omega_{i j}$ and $\Omega_{j i}=\Omega_{i j}$. Then $t_{i j}$ and $m_{i j}$ satisfy Eqs. (2.1).

In particular, if $c_{i}, \varphi_{i}, \theta_{i}, \phi_{i}$, and $\Omega_{i j}$ vanish identically, we recover Schaefer's [ 2 ] solution of the usual equations of continuum mechanics (cf. footnote in Section 4).

ThEorem 2.2. Let $\vartheta_{i}, \varphi_{i}$, and $\eta_{i}$ satisfy

$$
\begin{aligned}
\nabla^{2} \vartheta_{i}+e_{i j k} \varphi_{i, k} & =-f_{i}, \\
\nabla^{2} \eta_{i}+2 \varphi_{i} & =-c_{i} .
\end{aligned}
$$

Define $t_{i j}$ and $m_{i j}$ by

$$
\begin{aligned}
t_{i j} & =e_{i m p} e_{i n d} \omega_{m n, p q}-\delta_{i j} \vartheta_{k, k}+\vartheta_{i, i}+\vartheta_{i, i}+e_{i j k} \varphi_{k}, \\
m_{i j} & =e_{i m p} e_{i n Q} \chi_{m n, p q}-\delta_{i j} \sigma_{k, k}+\sigma_{i, i}+\eta_{i, i},
\end{aligned}
$$

where here $\omega_{j i}=\omega_{i j}$ but $\chi_{i j}$ need not be symmetric and $\sigma_{i}$ is arbitrary. Then $t_{i j}$ and $m_{i i}$ satisfy Eqs. (2.1).

The generality of the above solutions will be established in Section 4 .

3. Dipolar stresses. In the dipolar case of the multipolar continuum mechanics of Green and Rivlin [6], the equilibrium equations appear as

$$
\begin{aligned}
T_{i i, i}+F_{i} & =0, \\
M_{i\lfloor i k\}, i}+T_{\lfloor i k\}}+C_{[i k\}} & =0 .
\end{aligned}
$$

In Eqs. (3.1) $T_{i i}$ is the simple surface stress monopole, $M_{i i k}$ the simple surface stress dipole of the first kind, $F_{i}$ the simple body force monopole per unit volume, and $C_{i j}$ the simple body force dipole of the first kind per unit volume.

We note that, in light of Eqs. (2.4), Eqs. (3.1) have the same form as Eqs. (2.3). Hence for each of our results pertaining to $t_{i j}$ and $m_{i j k}$ an analogous result pertains to $T_{i j}$ and $M_{i[i k]}$. In particular, Eqs. (3.1) are equivalent to

$$
\begin{aligned}
T_{i j, i}+F_{i} & =0, \\
M_{i j, i}+e_{j k l} T_{k l}+C_{i} & =0,
\end{aligned}
$$

where

$$
\begin{aligned}
M_{i j} & =e_{i m n} M_{i m n} \quad\left(M_{i\lfloor i k\}}=\frac{1}{2} e_{l i k} M_{i l}\right), \\
C_{i} & =e_{i m n} C_{m n} \quad\left(C_{[i j]}=\frac{1}{2} e_{l i j} C_{l}\right) .
\end{aligned}
$$


The following theorem, which may be confirmed by substitution, provides an alternative solution in terms of $M_{i j k}$ and not just $M_{i[j k\}}$. It will be shown to be complete in Section 4 .

Theorem 3.1. Let $\vartheta_{i}, \varphi_{i}$, and $\psi_{i j}$ satisfy

$$
\begin{aligned}
\nabla^{2} \vartheta_{i}+e_{i j k} \varphi_{i, k} & =-F_{i}, \\
\nabla^{2} \psi_{[i i]}+e_{i i k} \varphi_{k} & =-C_{[i j]} .
\end{aligned}
$$

Define $T_{i j}$ and $M_{i j k}$ by

$$
\begin{aligned}
T_{i j}= & e_{i m p} e_{i n Q} \omega_{m n, p Q}-\delta_{i,} \vartheta_{k, k}+\vartheta_{i, j}+\vartheta_{i, i}+e_{i j k} \varphi_{k}, \\
M_{i j k}= & e_{i l p} e_{i m a} e_{k n r} \Lambda_{l m n, p a r}+\gamma_{l m, l m i j k}-\nabla^{2}\left(\gamma_{i l, l i k}+\gamma_{l i, l i k}\right)+\nabla^{4} \gamma_{i i, k} \\
& -\lambda_{l k, l i j}+\nabla^{2} \lambda_{i k, i}+\psi_{i k, i},
\end{aligned}
$$

where $\omega_{j i}=\omega_{i j}$ but $\Lambda_{i j k}, \gamma_{i j}$, and $\lambda_{i j}$ are arbitrary. Then $T_{i j}$ and $M_{i j k}$ satisfy Eqs. (3.1).

If we represent the skewsymmetric tensors $\psi_{[i i]}$ and $C_{[i i]}$ in terms of their dual vectors

$$
\begin{array}{ll}
\psi_{i}=e_{i m n} \psi_{m n} & \left(\psi_{[i i]}=\frac{1}{2} e_{l i i} \psi_{l}\right), \\
C_{i}=e_{i m n} C_{m n} & \left(C_{[i i]}=\frac{1}{2} e_{l i i} C_{l}\right),
\end{array}
$$

Eqs. (3.4) become

$$
\begin{aligned}
\nabla^{2} \vartheta_{i}+e_{i j k} \varphi_{i, k} & =-F_{i}, \\
\nabla^{2} \psi_{i}+2 \varphi_{i} & =-C_{i},
\end{aligned}
$$

and these equations are identical with Eqs. (2.7).

4. Completeness theorems. For the remainder of the paper $R$ will denote a bounded, open, integrable (Jordan-measurable) region of a three-dimensional Euclidean space. The closure of $R$ will be denoted by $R^{\prime}$. We write $f \varepsilon C^{N}(R)$ if and only if $f$ is a realvalued function defined and $N$ times continuously differentiable on $R$, and we write $f_{\varepsilon} C^{0}\left(R^{\prime}\right)$ if and only if $f$ is defined and continuous on $R^{\prime}$.

The proofs of our representation theorems will be based in part on the following lemmas. A proof of the first lemma can be found in Courant [4, p. 246]. The latter lemmas follow successively from the first one.

Lemma 4.1. Let $\mu \varepsilon C^{0}\left(R^{\prime}\right)$ and $\mu \varepsilon C^{N}(R)$ with $N \geq 1$. Define $f$ by

$$
f(\mathrm{x})=-\frac{1}{4 \pi} \int_{R} \frac{\mu(\xi)}{|\mathrm{x}-\xi|} d \xi .
$$

Then $f \varepsilon C^{N+1}(R)$ and $\nabla^{2} f=\mu$ on $R$.

Lemma 4.2. Let the hypotheses of Lemma 4.1 hold but with $N \geq 2$, and define f by (4.1). Define $g$ by

$$
g(\mathrm{x})=-\frac{1}{4 \pi} \int_{R} \frac{f(\xi)}{|\mathrm{x}-\xi|} d \xi .
$$

Then $g \varepsilon C^{N+2}(R)$ and $\nabla^{4} g=\mu$ on $R$.

Lemma 4.3. Let the hypotheses of Lemma 4.1 hold but with $N \geq 3$, and define $f$ and $g$ 
by (4.1) and (4.2), respectively. Define $h$ by

$$
h(\mathbf{x})=-\frac{1}{4 \pi} \int_{R} \frac{g(\xi)}{|\mathbf{x}-\xi|} d \xi .
$$

Then $h \varepsilon C^{N+3}(R)$ and $\nabla^{6} h=\mu$ on $R$.

We now turn to the statements and proofs of our completeness theorems.

THEOREM 4.1. Let $t_{(i j)} \varepsilon C^{0}\left(R^{\prime}\right), t_{(i j)} \varepsilon C^{N}(R)$, and $m_{(i i)} \varepsilon C^{0}\left(R^{\prime}\right), m_{(i i)} \varepsilon C^{N}(R)$ with $N \geq 2$. Then there exist $\omega_{i j}=\omega_{i i} \varepsilon C^{N}(R), \vartheta_{i} \varepsilon C^{N-1}(R), \varphi_{i}$, and $\Omega_{i j}=\Omega_{i i} \varepsilon C^{N}(R)$, $\theta_{i} \varepsilon C^{N-1}(R), \phi_{i}$ such that

$$
t_{i j}=e_{i m p} e_{i n q} \omega_{m n, p a}-\delta_{i j} \vartheta_{k, k}+\vartheta_{i, j}+\vartheta_{j, i}+e_{i j k} \varphi_{k}
$$

and

$$
m_{i j}=e_{i m p} e_{i n q} \Omega_{m n, p q}-\delta_{i j} \theta_{k, k}+\theta_{i, j}+\theta_{i, i}+e_{i j k} \phi_{k} .
$$

Moreover, if $N \geq 3, t_{\{i j\}} \varepsilon C^{1}(R), m_{\{i j\}} \varepsilon C^{1}(R)$, and $t_{i j}$ and $m_{i j}$ satisfy Eqs. (2.1); then

$$
\begin{aligned}
\nabla^{2} \vartheta_{i}+e_{i j k} \varphi_{i, k} & =-f_{i}, \\
\nabla^{2} \theta_{i}+e_{i j k} \phi_{i, k}+2 \varphi_{i} & =-c_{i} .
\end{aligned}
$$

Proof. The second part of the theorem follows from Theorem 2.1. Since the representations of $t_{i j}$ and $m_{i j}$ are of the same form, it suffices to establish representation (4.4) for $t_{i j}$. We decompose $t_{i j}$ into its symmetric and skewsymmetric parts:

$$
t_{i i}=t_{(i j)}+t_{(i j)} .
$$

Setting

$$
\varphi_{i}=\frac{1}{2} e_{i m n} t_{m n}
$$

we have

$$
t_{(i j)}=e_{i j k} \varphi_{k} .
$$

Turning now to the representation of $t_{(i i)}$, we note that by Lemma 4.2 there exist $A_{i j}=A_{i i} \varepsilon C^{N+2}(R)$ such that

$$
t_{(i i)}=\nabla^{4} A_{i j}
$$

Also, we have the identity

$$
\nabla^{4} A_{i j}=e_{i l a} e_{j m l} e_{l p c} e_{m a l} A_{p a, a b c d}-A_{l m, l m i j}+\nabla^{2}\left(A_{i l, l j}+A_{l i, l i}\right),
$$

as is readily verified with the aid of

$$
e_{i j k} e_{i p q}=\delta_{j p} \delta_{k q}-\delta_{i q} \delta_{k p}
$$

Next define

$$
\begin{aligned}
\omega_{l m}^{\prime} & =e_{l p c} e_{m a d} A_{p q, c d}, \\
\vartheta_{i}^{\prime} & =A_{i l, l},
\end{aligned}
$$

and conclude from Eqs. (4.10) and (4.11) and the symmetry of $A_{i j}$ that

$$
t_{(i j)}=e_{i m p} e_{i n q} \omega_{m n, p q}^{\prime}-\vartheta_{k, k i j}^{\prime}+\nabla^{2}\left(\vartheta_{i, j}^{\prime}+\vartheta_{j, i}^{\prime}\right) \text {. }
$$


Now set

$$
\Gamma_{i j}=\delta_{i ;} \vartheta_{k, k}^{\prime}
$$

and note that

$$
e_{i m p} e_{i n q} \Gamma_{m n, p q}=\delta_{i j} \nabla^{2} \vartheta_{k, k}^{\prime}-\vartheta_{k, k i j}^{\prime} .
$$

Eqs. (4.14) and (4.16) yield

$$
t_{(i i)}=e_{i m p} e_{i n q}\left(\omega_{m n, p q}^{\prime}+\Omega_{m n, p q}\right)-\delta_{i i} \nabla^{2} \vartheta_{k, k}^{\prime}+\nabla^{2}\left(\vartheta_{i, i}^{\prime}+\vartheta_{i, i}^{\prime}\right) .
$$

The jroof is completed by defining

$$
\begin{aligned}
\omega_{i j} & =\omega_{i j}^{\prime}+\Gamma_{i i}, \\
\vartheta_{i} & =\nabla^{2} \vartheta_{i}^{\prime},
\end{aligned}
$$

so that

$$
t_{(i j)}=e_{i m p} e_{i n q} \omega_{m n, p q}-\delta_{i j} \vartheta_{k, k}+\vartheta_{i, i}+\vartheta_{i, i} .
$$

The representation (4.14) of the symmetric tensor $t_{(i i)}$ is due to Gurtin [5] as is the step which leads to the simpler representation (4.19).*

THEOREM 4.2. Let $t_{(i i)} \varepsilon C^{0}\left(R^{\prime}\right), t_{(i i)} \varepsilon C^{N}(R)$, and $m_{i j} \varepsilon C^{0}\left(R^{\prime}\right), m_{i j} \varepsilon C^{N}(R)$ with $N \geq 2$. Then there exist $\omega_{i i}=\omega_{i i} \varepsilon C^{N}(R), \vartheta_{i} \varepsilon C^{N-1}(R), \varphi_{i}$, and $\chi_{i j} \varepsilon C^{N}(R), \sigma_{i} \varepsilon C^{N-1}(R)$, $\eta_{i} \varepsilon C^{N-1}(R)$ such that

$$
t_{i j}=e_{i m p} e_{i n q} \omega_{m n, p q}-\delta_{i j} \vartheta_{k, k}+\vartheta_{i, i}+\vartheta_{i, i}+e_{i j k} \varphi_{k}
$$

and

$$
m_{i j}=e_{i m p} e_{i n q} \chi_{m n, p q}-\delta_{i j} \sigma_{k, k}+\sigma_{i, i}+\eta_{i, i} .
$$

Morewer, if $N \geq 3, t_{[i j]} \varepsilon C^{1}(R)$, and $t_{i j}$ and $m_{i j}$ meet (2.1); then

$$
\begin{aligned}
\nabla^{2} \vartheta_{i}+e_{i j k} \varphi_{j, k} & =-f_{i}, \\
\nabla^{2} \eta_{i}+2 \varphi_{i} & =-c_{i} .
\end{aligned}
$$

Proof. The second part of the theorem follows from Theorem 2.2, and the representation (4.20) of $t_{i j}$ was established in Theorem 4.1. We are left with verifying the representation (4.21) of $m_{i j}$. By Lemma 4.2 there exist $B_{i j} \varepsilon C^{N+2}(R)$ such that

$$
m_{i j}=\nabla^{4} B_{i j} .
$$

*Representation (4.19) gives us a refinement of Gurtin's [5] generalized Beltrami solution of the usual equations of continuum mechanics. If

$$
\tau_{i j}=e_{i m p} e_{j n q} \omega_{m n, p q}-\delta_{i j} \vartheta_{k, k}+\vartheta_{i, j}+\vartheta_{i, i} \quad\left(\omega_{j i}=\omega_{i j}\right)
$$

and $\nabla^{2} \vartheta_{i}=-b_{i}$, then $\tau_{i j}$ is a solution of

Setting

$$
\tau_{i j, i}+b_{j}=0, \quad \tau_{j i}=\tau_{i j} .
$$

$$
\Sigma_{i j}=\omega_{i j}-\frac{1}{2} \delta_{i j} \omega_{k k} \quad\left(\omega_{i j}=\Sigma_{i j}-\delta_{i j} \Sigma_{k k}\right)
$$

we obtain Schaefer's [2] solution

$$
\tau_{i j}=-\nabla^{2} \Sigma_{i j}+\Sigma_{i m, m i}+\Sigma_{j m, m i}-\delta_{i j} \Sigma_{m n, m n}-\delta_{i j} \vartheta_{k, k}+\vartheta_{i, j}+\vartheta_{i, i}
$$


Again we have the identity

Defining

$$
\nabla^{4} B_{i j}=e_{i l a} e_{j m b} e_{l p c} e_{m a d} B_{p q, a b c d}-B_{l m, l m i j}+\nabla^{2}\left(B_{i l, l j}+B_{l i, l i}\right) .
$$

$$
\begin{aligned}
\chi_{l m}^{\prime} & =e_{l p c} e_{m a d} B_{p a, c d}, \\
\sigma_{i}^{\prime} & =B_{i l, l}, \\
\eta_{i} & =\nabla^{2} B_{l i, l},
\end{aligned}
$$

we have from Eqs. (4.23) and (4.24) that

Setting

$$
m_{i j}=e_{i m p} e_{i n q} \chi_{m n, p q}^{\prime}-\sigma_{k, k i i}^{\prime}+\nabla^{2} \sigma_{i, i}^{\prime}+\eta_{i, i}
$$

completes the proof.

$$
\begin{aligned}
\chi_{i i} & =\chi_{i j}^{\prime}+\delta_{i j} \sigma_{k, k}^{\prime}, \\
\sigma_{i} & =\nabla^{2} \sigma_{i}^{\prime}
\end{aligned}
$$

TheOREM 4.3. Let $T_{(i i)} \varepsilon C^{0}\left(R^{\prime}\right), T_{(i i)} \varepsilon C^{N}(R)$, and $M_{i j k} \varepsilon C^{0}\left(R^{\prime}\right), M_{i i k} \varepsilon C^{N+1}(R)$ with $N \geq 2$. Then there exist $\omega_{i j}=\omega_{i i} \varepsilon C^{N}(R), \vartheta_{i} \varepsilon C^{N-1}(R), \varphi_{i}$, and $\Lambda_{i i k} \varepsilon C^{N+1}(R)$, $\gamma_{i j} \varepsilon C^{N+3}(R), \lambda_{i j} \varepsilon C^{N+1}(R), \psi_{i j} \varepsilon C^{N-1}(R)$ such that

$$
T_{i j}=e_{i m p} e_{i n Q} \omega_{m n, p q}-\delta_{i j} \vartheta_{k, k}+\vartheta_{i, j}+\vartheta_{i, i}+e_{i j k} \varphi_{k}
$$

and

$$
\begin{array}{r}
M_{i j k}=e_{i l p} e_{j m Q} e_{k n r} \Lambda_{l m n, p q r}+\gamma_{l m, l m i j k}-\nabla^{2}\left(\gamma_{i l, l i k}+\gamma_{l i, l i k}\right)+\nabla^{4} \gamma_{i j, k} \\
-\lambda_{l k, l i j}+\nabla^{2} \lambda_{i k, i}+\psi_{i k, i} .
\end{array}
$$

Moreover, if $N \geq 3, T_{(i, i]} \varepsilon C^{1}(R)$, and $T_{i j}$ and $M_{i j k}$ satisfy Eqs. (3.1); then

$$
\begin{aligned}
\nabla^{2} \vartheta_{i}+e_{i i k} \varphi_{i, k} & =-F_{i}, \\
\nabla^{2} \psi_{[i i]}+e_{i j k} \varphi_{k} & =-C_{[i i]} .
\end{aligned}
$$

Proof. The second part of the theorem follows from Theorem 3.1, and the representation (4.28) of $T_{i i}$ was established in Theorem 4.1. The representation (4.29) of $M_{i j k}$ is deduced as follows. By Lemma 4.3 there exist $C_{i j k} \varepsilon C^{N+4}(R)$ such that

$$
M_{i j k}=\nabla^{6} C_{i j k} \text {. }
$$

Also, in view of (4.12), we have the identity

$$
\begin{aligned}
& \nabla^{6} C_{i j k}=-e_{i l a} e_{i m b} e_{k n e} e_{l p d} e_{m e e} e_{n r} C_{p a r, a b c d e s}+C_{l m n, l m n i j k} \\
& \quad-\nabla^{2}\left(C_{i l m, l m i k}+C_{l i m, l m i k}+C_{l m k, l m i i}\right)+\nabla^{4}\left(C_{l i k, l i}+C_{i l k, l j}+C_{i j l, l k}\right) .
\end{aligned}
$$

Now define

$$
\begin{aligned}
\Lambda_{l m n} & =-e_{l p d} e_{m a e} e_{n r} C_{p e r, d e f} \\
\gamma_{i i} & =C_{i i l, l} \\
\lambda_{i j} & =\nabla^{2} C_{i l i, l} \\
\psi_{i j} & =\nabla^{4} C_{l i i, l}
\end{aligned}
$$

and the theorem is proved. 
Acknowledgement. The author is indebted to M. E. Gurtin for suggesting this investigation and for valuable discussions.

\section{REFERENCES}

1. E. and F. Cosserat, Théorie des Corps Déformables, A. Hermann, Paris, 1909

2. H. Schaefer, Die Spannungsfunktionen des dreidimensionalen Kontinuums und des elastischen Körpers, Z. Angew. Math. Mech. 33 (1953) 356-362

3. W. Günther, Zur Statik und Kinematik des Cosseratschen Kontinuums, Abh. Braunschweig. Wiss. Ges. 10 (1958), 195-213

4. R. Courant, Partial differential equations (Vol. 2 of Methods of mathematical physics by R. Courant and D. Hilbert), Interscience, New York, 1962

5. M. E. Gurtin, A generalization of the Beltrami stress functions in continuum mechanics, Arch. Rat. Mech. Anal. 13, 5 (1963), 321-329

6. A. E. Green and R. S. Rivlin, Simple force and stress multipoles, Arch. Rat. Mech. Anal. 16 (1964), 5, 325-353 